\title{
Colorimetric Assessment of Surface Sealants for Discoloration of a Nanofilled Resin Composite
}

\author{
Bora Korkut iD \\ Marmara University, Faculty of Dentistry, Department of Restorative Dentistry, Istanbul, Turkey. \\ Correspondence Author: Bora Korkut \\ E-mail: bora.korkut@marmara.edu.tr \\ Received: $10.10 .2020 \quad$ Accepted: 30.11 .2020
}

\begin{abstract}
Objective: This in vitro study was aimed to assess the effect of different surface sealants on discoloration of a nonofilled resin-based composite quantitatively, using a colorimeter.

Methods: 40 specimens were prepared using nanofilled resin composite, Filtek Universal Restorative (A2 shade, 3M, USA). Sprecimens were polymerized from both sides, polished using polishing discs (SofLex, 3M, USA) and divided by test groups ( $n=10$ for each), regarding the sealant used; Permaseal (Ultradent Products, USA), Biscover LV (Bisco, USA), Optiglaze Clear (GC Corp., Japan) and control group. Samples were discolored for 144 hours in coffee solution, and color measurements were performed using colorimeter (ShadeStar, Dentsply Sirona, USA). Nominal color codes of specimens regarding Vita Classical Shade Guide, were converted to corresponding numerical values. Level of color change after sealant application ( $\Delta$ Vita1) and after discoloration ( $\Delta$ Vita2) were calculated. Shapiro Wilk and Kruskal Wallis tests were used for statistical analyses.

Results: Color changes in Permaseal, Optiglaze and control groups were significant $(p<0.001)$ compared to Biscover, for $\Delta$ Vita1 scores. Remarkable level of darkening was observed for all groups, for $\Delta$ Vita2 scores. Permaseal revealed significantly the highest level of darkening, among all $(p<0.001)$, but no difference with control group. Optiglaze showed significantly lower level of darkening compared to the control group ( $p<0.001)$, whereas no difference with Biscover $(p \geq 0.05)$. Biscover group showed similar level of darkening with Optiglaze and control groups $(p \geq 0.05)$.

Conclusion: Permaseal sealant presented significantly the highest discoloration, whereas Biscover and Optiglaze sealants presented similar and lower. Colorimeter might be determined as repeatable method for measuring discoloration in vitro.

Keywords: Sealant, Biscover, Optiglaze, Permaseal, discoloration, nanocomposite
\end{abstract}

\section{Clinical relevance statement:}

Discoloration of resin-based composite restorations may cause esthetic problems clinically, in long-term clinical. The use of surface sealants might be a supporting procedure for maintaining the color strability of composite restorations. In this study, the effect of various surface sealants with a nanofilled composite was assessed in vitro, regarding corresponding coffee discoloration for 6 months.

\section{INTRODUCTION}

Resin-based composite (RBC) materials have been preferred frequently for direct anterior restorations, with the increasing expectations in esthetics (1). Accordingly, surface characteristics of the RBC restoration plays a key role in longterm esthetic outcome. Polishing capacity of the composite material, surface hardness and surface roughness are the important factors influencing the the color stability (2). Resin matrix absorbs water to compensate polymerization shrinkage $(1,2)$. Unreacted free monomers may develop due to the insufficient polymerization rate and may increase solubility of the resin, microleakage and thereby potentially discoloration as a result of decrease in mechanical properties (2). Etiology of discoloration may be related to internal and external factors. Discoloration is not only related to to the polymerization level, but also the inorganic filler content of the material and deficiencies in polishing procedures $(3,4)$. Type, shape, size, and amount of the inorganic filler particles may alter the physical properties of the RBC material. Also effective polishing using proper polishing materials is essential to achieve a smooth restoration surface, thereby, inhibiting the plaque accumulation and the caries process (5). Whereas, patient related factors (i.e., systemic diseases, 
oral hygiene, diet, and smoking habits) may also influence the long-term color stability of RBC restorations.

Surface sealants are resin-based materials developed to maintain the color stability of the RBC restorations in longterm. The low-viscosity structure led these materials to cover the surface of restorations by penetrating through microporosities. Aim of surface sealants is to cover the surface permanently as a thin film layer, reduce the water absorption of the material, and thereby maintaining the color stability $(1,6,7)$. Previous studies regarding the effectiveness of surface sealants have been controversial. Some researchers suggested the application, whereas some reported no meaning in application (6-8). There are a few surface sealants of different brands available in the market currently and each have individual application protocols.

Various diagnostic techniques have been used to evaluate the color stability of RBCs. Colorimeters, spectrophotometers, and recently cross-polarization dental photography are quantitative techniques which were mentioned previously in literature for the color assessment (9).

Quantitative scoring of color change have frequently been performed according to Comission Internationale de I'Eclairage (CIE) color universe (9-11), which includes mainly two color coordinate systems, $L^{*} a^{*} b^{*}$ and $L^{*} C^{*} h^{*}$. Color is expressed by three parameters in $L^{*} a^{*} b^{*}$ coordinate system. $L^{*}$ refers to brightness between black $(0)$ and white (100), a* refers to color from red $\left(+a^{*}\right)$ to yellow $\left(-a^{*}\right), b^{*}$ refers to the color from yellow $\left(+b^{*}\right)$ to blue $\left(-b^{*}\right) .{ }^{9,10} L^{*} c^{*} h^{*}$ coordinate system was reported to be more compatible with human eye regarding the color perception. $L^{*}$ refers to luminance, $C^{*}$ refers to chroma, and $h^{*}$ refers to hue (10). In addition, another color system, CIEDE2000 $\left(\Delta \mathrm{E}_{00}\right)$, was introduced to improve the performance of gray and blue colors, recently (12). However, controversial results are available in literature, regarding the sensitivity of CIEDE2000 system $(11,12)$.

The objective of this in vitro study was to assess the effect of different surface sealants on discoloration of a contemporary nonofilled RBC quantitatively, using a colorimeter. The null $\left(h_{0}\right)$ hypothesis was determined as follows; the use of surface sealants had no effect on the level of discoloration of nanofilled composites.

\section{METHODS}

A novel nanofilled RBC, Filtek Universal Restorative (3M, MN, USA) was used in this study. A total of 40 disc-shaped specimens of $A 2$ shade were prepared. Disc molds of $12 \mathrm{~mm}$ in diameter and $2 \mathrm{~mm}$ thickness were used for the preparation. A polywave LED unit (Valo Grand, Ultradent Products, USA) with $12 \mathrm{~mm}$ tip in diameter was used for polymerization of the samples. The curing unit was calibrated with a radiometer before each cure. The specimens were polymerized from both sides for $20 \mathrm{~s}$, an irradiance of $1000 \mathrm{mw} / \mathrm{cm}^{2}$. Surface abrasions were performed for $20 \mathrm{~s}$ on both surfaces, using 600 grit silicone carbide ( $\mathrm{SiC}$ ) abrasion papers (30 $\mu \mathrm{m}$ abrasive particle size), for initial surface standardization. A new abrasion paper was used per specimen. Following the preparation, the specimens were kept in distilled water for one day, at room temperature.

Three different resin-based surface sealant agents were used in this study; Permaseal (Ultradent Products, USA), Biscover LV (Bisco, USA), Optiglaze Color Clear (GC Corp., Japan). The specimens were divided into 4 groups (i.e., Permaseal group $(n=10)$; Biscover group $(n=10)$; Optiglaze group $(n=$ $10)$, and control group ( $n=10)$. All specimens including the control group were polished from both sides, using Sof-Lex polishing discs ( $3 \mathrm{M}$, St. Paul, MN, USA) in four different grains (i.e., thick, $80 \mu$; medium, $40 \mu$; fine, $24 \mu$; ultrafine, $8 \mu$ ). New discs were used per specimen for $30 \mathrm{~s}$, under dry conditions, at $10000 \mathrm{rpm}$ and the surfaces of the specimens were gently cleaned from debris before changing the disc.

Surface sealants were applied on polished surfaces of the specimens. No surface sealant agent was applied to the specimens in control group. The sealant agents were used according to the individual manufacturers' instructions. Polymerization of the sealants were also performed using the same LED unit at an irradiance of $1000 \mathrm{~mW} / \mathrm{cm}^{2}$. The contents and composition of the composite material and the sealant agents were resented in Table 1.

Phosphoric acid was used on sample surfaces prior to the application of Biscover LV and Permaseal sealants according to the manufacturers' instructions, to clean the surface of resin composite.

Regarding Permaseal application, $37 \%$ phosphoric acid was applied on polished surfaces for $20 \mathrm{~s}$, the sealant was applied on both sides, dried with slight air for $5 \mathrm{~s}$, and then polymerized for $20 \mathrm{~s}$ from both sides.

Regarding Biscover LV application, 37 \% phosphoric acid was applied on polished surfaces for $30 \mathrm{~s}$, the sealant was applied on both sides, slightly refined with air, and then polymerized for $20 \mathrm{~s}$ from both sides.

Regarding Optiglaze Color Clear application, composite primer and sealant agent were applied on both sides, respectively. The surfaces were polymerized for $20 \mathrm{~s}$ from both sides.

Following the sealant application on polished surfaces, all specimens were slightly cleaned from debris with continuous water and stored in distilled water for a day. Then the samples were immersed in staining solution, inside an incubator, at constant room temperature for 144 hours, which corresponds to 6 months of coffee consumption $(13,14)$. The staining solution was prepared by dissolving 20 gr of coffee (Nescafe Gold, Nestle SA, Switzerland) in $250 \mathrm{ml}$ of $100{ }^{\circ} \mathrm{C}$ boiling water (14), and was renewed daily. At the end of discoloration procedure, the specimens were rinsed and gently dried.

The color of the specimens was measured using a clinical type colorimeter (ShadeStar, Dentsply Sirona, USA) with Vita Shade Guide mode. Colorimeter was calibrated before each measurement using the calibration unit on individual stand 
of the device. Specimens were gently dried using a tissue paper before the color measurements. The Vita Classic shade of each specimen was collected for the baseline $\left(T_{1}\right)$, after sealant application $\left(T_{2}\right)$ and after discoloration precedure $\left(T_{3}\right)$. The collected nominal color codes form B1 to D4 were converted to numerical values of $1-16$, in accordance to the Vita Classic Shade Guide Scale. The level of potential color change for each specimen after sealant application was assessed by calculating the difference between $T_{2}-T_{1}$
$\left(\Delta \mathrm{Vita}_{1}\right)$. The level of discoloration was assessed by calculating the difference between $\mathrm{T}_{3}-\mathrm{T}_{2}\left(\Delta \mathrm{Vita}_{2}\right)$.

Statistical analysis was performed using IBM SPSS V23 sofware. The normality of the data was investigated by using Shapiro Wilk test. Comparisons between the groups were investigated by using Kruskal Wallis test and the results of the analysis were presented as medium (min-max). ' $p$ ' value of .05 was deemed significant.

Table 1. Compositions, type and manufacturers of the resin composites and the surface sealants tested in this study.

\begin{tabular}{|l|l|l|l|l|l|}
\hline $\begin{array}{l}\text { Resin-based } \\
\text { material }\end{array}$ & Shade & Filler & Composition & Manufacturer \\
\hline $\begin{array}{l}\text { Filtek Universal } \\
\text { Restorative }\end{array}$ & A2 enamel & Nanofilled & $\begin{array}{l}\text { Matrix: Bis-GMA, UDMA, TEGDMA, PEGDMA, Bis-EMA } \\
\frac{\text { Filler: Silica filler (20nm), zirconia filler (4-11nm), zirconia/silica cluster filler. 0,6-10 microns }}{\text { particle size. 78.5 wt\%, 63.5 vol\% }}\end{array}$ & $\begin{array}{l}3 \mathrm{M}, \text { St. Paul, MN, } \\
\text { USA }\end{array}$ \\
\hline Permaseal & - & Unfilled & BIS-GMA 60\%, TEGDMA 40\%, 1-dimethylaminoethyl metacrylate <3\% & $\begin{array}{l}\text { Ultradent } \\
\text { Products, South } \\
\text { Jordan, UT, USA }\end{array}$ \\
\hline $\begin{array}{l}\text { Biscover } \\
\text { LV }\end{array}$ & - & Unfilled & Dipentaerythritol penta-acrylateesters and ethanol & $\begin{array}{l}\text { Bisco Inc., } \\
\text { Schaumburg, IL, } \\
\text { USA }\end{array}$ \\
\hline $\begin{array}{l}\text { Optiglaze Color } \\
\text { Clear }\end{array}$ & - & Nanofilled & $\begin{array}{l}\text { Methyl-methacrylate (30-40\%), Silica filler(10\%), } \\
\text { Multifunctional acrylate(50-60\%), diphenyl(2,4,6-trimethylbenzoyl)-phosphine oxide(less } \\
\text { than5\%), Photoinitator }\end{array}$ & $\begin{array}{l}\text { GC Corp., Tokyo, } \\
\text { Japan }\end{array}$ \\
\hline
\end{tabular}

Bis-EMA=ethoxylated bisphenol-A dimethacrylate; Bis-GMA=bisphenol-glycidyl methacrylate; TEGDMA=triethyleneglycol dimethacrylate; UDMA=urethane dimethacrylate; PEGDMA=polyethylene glycol dimethacrylate.

*The data regarding the compositions of theRBCs were obtained from the manufacturers of the composites.

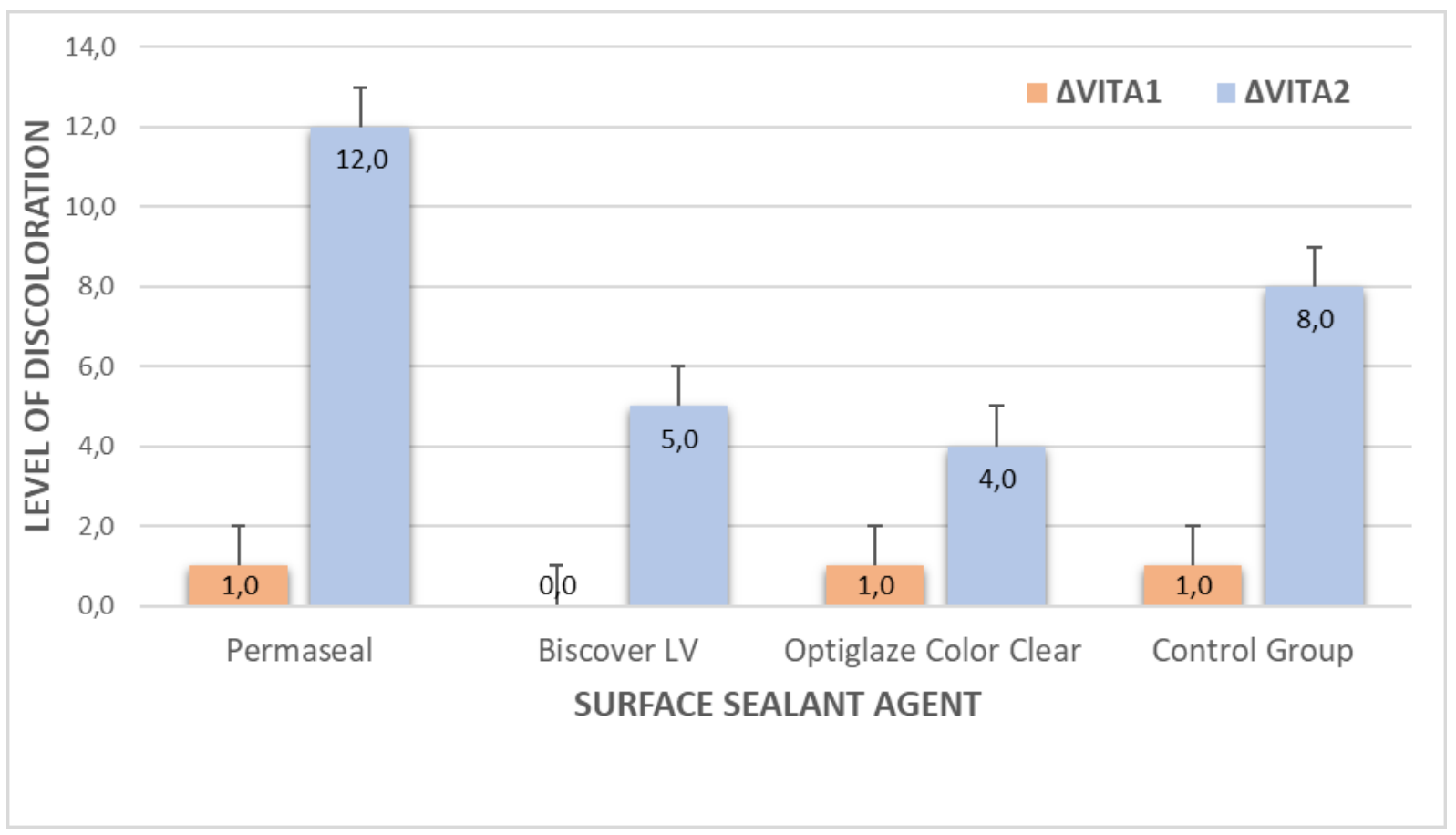

Figure 1. The level of discoloration for surface sealant agents, regarding $\Delta V$ Vita1 and $\Delta V$ Vita2 scores. 


\section{RESULTS}

A good intra-observer agreement was obtained regarding the kappa values of 0.92 and 0.94 . Initial color of the specimens were ranged between $B 2$ to $D 2$, and no significant changes in color were observed for Biscover group at $T_{1}, T_{2}$, and $T_{3^{\prime}}$ in terms of $\Delta \mathrm{Vita}_{1}$ scores. However, significant color changes were observed for the specimens in Permaseal, Optiglaze and control groups compared to Biscover group ( $p<0.001)$. Level of color changes in Permaseal, Optiglaze and control groups were only 1 unit, and statistically similar ( $p \geq 0.05$; Table 2).

Table 2. Comparisons between the surface sealant agents, regarding VITA1 and $\triangle$ VITA2 scores

\begin{tabular}{lll} 
& $\Delta$ VITA $_{1}$ & $\Delta$ VITA $_{2}$ \\
\hline Permaseal & $1(0-1)^{\mathrm{a}}$ & $12\left(8-13^{)} \mathrm{c}\right.$ \\
Biscover LV & $0(0-1)^{\mathrm{b}}$ & $5(4-5)^{\mathrm{ab}}$ \\
Optiglaze Color Clear & $1(0-1)^{\mathrm{a}}$ & $4(2-5)^{\mathrm{b}}$ \\
Control Group & $1(0-1)^{\mathrm{a}}$ & $8(7-8)^{\mathrm{ac}}$ \\
p $^{*}$ & $<0.001$ & $<0.001$ \\
\hline
\end{tabular}

${ }^{*} a-c$ : There is no difference between the groups with the same letter, for each column.

Remarkable level of darkening in color was observed for all specimens after coffee discoloration for 144 hours, in terms of $\Delta V_{i t a}$ scores (Graphic 1). Permaseal group revealed significantly the highest level of darkening, among others $(p<$ 0.001 ), but no significant difference was observed with control group ( $p \geq 0.05$ ). Optiglaze group showed significantly lower level of darkening than control group $(p<0.001)$, whereas no significant diffrerence was observed with Biscover $(p \geq 0.05)$. Biscover and control groups revealed statistically similar level of discolorations ( $p \geq 0.05$; Table 2).

\section{DISCUSSION}

The null hypothesis $\left(h_{0}\right)$ was rejected, because the surface covering agents have affected the level of discoloration of the composite material used.

Flexible aluminum oxide discs were used for polishing in this study, which were considered gold standard materials for obtaining the highest surface smoothness on composite surface (15-18). It was aimed to obtain the optimum surface standardization by using the polishing system in four grains, and also to increase the accuracy of color measurements by eliminating the microgap formations on surfaces $(14,19)$.

Coffee was used as staining solution in this study to simulate daily routine in vitro, with regards to the previously reported high effectiveness in discoloration of RBCs, because of the yellow colorants $(19,20)$. The absorbtion and adsorbtion of colorants through the organic phase was described as staining mechanism of the coffee (20) and high discoloration of resins was reported after storage in coffee for two days period, previously (21). It was determined as the most effective agent for discoloration of RBCs (22). Mundim et al. (20) reported 15 days of storage in coffee simulated the 1 year consumption. Rajkumar et al. (14) reported $15 \mathrm{~min}$ as the average consumption time of a cup of coffee and 3,2 cups per day. With regards to the previous results, the specimens were kept in coffee solution at room temperature for 144 hours, corresponding to the consumption of 6 months $(14,21)$.

A contemporary nanofilled RBC was used in this study for all sealant groups. Less color change was reported to be expected for nanofilled composites, because of presenting smoother surfaces with less stains, as a result of smaller filler size $(16,22-24)$. The inorganic filler content is silica, zirconia and silica/zirconia cluster fillers with $78,5 \mathrm{wt} \%$ and 63,5 vol $\%$ (Table 1). Also triethyleneglycol dimethacrylate (TEGDMA) has enhancing effect in degree of polymerization, elastic modulus, and surface hardness $(18,24,25)$, and urethane dimethacrylate (UDMA) has lower rate of water absorption $(14,17)$. Regarding our results, these resins and hard filler particles in high concentration might have influenced the resistance to discoloration of the specimens. Whereas, only a single RBC was used in total and thereby resin composite was not an effective factor on color change for this study.

Repeatability of colorimeter measurements were confirmed by obtaining good intra-observer agreement ( $k=0.92$ and 0.94). All surface sealants were polymerized for $40 \mathrm{~s}$ in our study, therefore curing time might not be considered an effective factor. Although all specimens were produced using A2 shade of Filtek Universal Restorative (3M), the measured colors were B2 or D2, which were 2 and 1 unit(s) different, respectively, according to the Vita Classic Shade Guide Scale. However, as the statistical analyses were performed according to the color differences ( $\Delta$ Vita scores), standardization in color initially was not mandatory for this study.

Regarding the $\Delta$ Vita $_{1}$ scores, all groups showed significant color changes after surface sealant application except Biscover group, which showed only one unit of discoloration, according to the Vita Classic Shade Guide Scale (Table 2). The surface gloss obtained after surface polishing might be responsible for this slight color change, as light reflections might create deceiving effect on surface during the color assessment (10). However, no color change was observed for Biscover group which might be interpreted that, Biscover sealant might have prevented the unwilling reflection effect of polishing, whereas Permaseal and Optiglaze sealants could not. Therefore, with regards to our results, surface polishing as well as surface gloss might be considered effecting factors on digital color measurement of RBCs.

Regarding the $\Delta \mathrm{Vita}_{2}$ scores, the specimens in all groups were discolored significantly after coffee discoloration for 144 hours (Graphic 1). Coffee was considered an effective agent on discoloration of nanofilled RBC, supporting the previous results (23). The specimens in Permaseal group [12 $\left.(8-13)^{\mathrm{c}}\right]$ and control group [8 $(7-8)^{\text {ac }}$ ] statistically discolored similarly, however, Permaseal showed a greater 
level of discoloration mathematically (Table 1). Permaseal also showed the greatest level of discoloration among other sealants. Permaseal contains bisphenol-glycidyl methacrylate (Bis-GMA) resin and more staining potential was reported for the RBCs including (Bis-GMA) resin than UDMA, previously $(14,17)$. Furthermore, hydrophilic TEGDMA resin might have also increased the water uptake of the Bis-GMA based Permaseal (25) and thereby, both factors potentially affected the greater staining in coffee solution (26).

In the present study, the specimens in Optiglaze [4 (2 - 5) $\left.{ }^{\mathrm{b}}\right]$ and Biscover [5 $\left.(4-5)^{\mathrm{ab}}\right]$ groups showed similar and the lowest level of discolorations mathematically (Graphic 1). The color stability of the specimens in Optiglaze group was significantly better than control group. In addition, Biscover group showed similar level of discoloration with Optiglaze and control groups (Table 2). With regards to these results, Optiglaze might have revealed a slightly better performance compared to Biscover, although no significant difference was determined between. Thus, our study agreed the results of Sahin et al. (26), who reported Biscover less stain resistant than Optiglaze. On the contrary, our results have conflicted the previous results of that, Optiglaze could cause increase staining because of debonding of nanofillers form resin matrix and thereby, forming void formations on the surface $(17,27)$. However, positive effects of nanofillers on prevention of discoloration were also reported (23).

In this study, all surface sealants were applied on wellpolished resin composite surfaces, which might have caused debonding problems during discoloration process (28). This prespective should be considered for further studies. Only one type of resin composite and staining solution were used which might also be interpreted as a limitation of this study. Also, clinical factors such as occlusal relations, the effect of saliva, diet, and toothbrushing, should be taken into consideration. Therefore, the conclusions of this study should be verified with further clinical trials.

\section{CONCLUSION}

Within the limitations of this in vitro study, the experimented surface sealants might be considered effective on discoloration of the nanofilled composite, in terms of coffee discoloration for six months. Specimens with Permaseal sealant showed the highest discoloration, significantly. Specimens with Biscover LV and Optiglaze Color Clear sealants showed similar and lower level of discolorations. Colorimeter might be determined as a repeatable method for measuring the level of discoloration in vitro. The conclusions of this study should be verified with different staining solutions, RBCs and further clinical trials.

\section{Conflict of Interest}

The manuscript has been read and approved by all the authors. No potential conflict of interest was reported by any of the authors in this study.

\section{REFERENCES}

[1] Khalaj K, Soudi A, Tayefi-Nasrabadi M, Keshvad, M. The evaluation of surface sealants' effect on the color stability of Nano-hybrid composite after polishing with One-Step system (in-vitro). J Clin Exp Dent 2018;10(9):927-932.

[2] Beltrami R, Ceci M, De Pani G, Vialba L, Frederico R, Poggio C, Colombo M. Effect of different surface finishing/polishing procedures on color stability of esthetic restorative materials: a spectrophotometric evaluation. Eur J Dent 2018;12(1):49-56.

[3] Poggio C, Ceci M, Beltrami R, Mirando M, Wassim J, Colombo M. Color stability of esthetic restorative materials: a spectrophotometric analysis. Acta Biomater Odontol Scand 2016;2(1):95-101.

[4] Halacoglu D, Yamanel K, Basaran S, Tuncer D, Celik C. Effects of staining and bleaching on a nano-hybrid composite with or without surface sealant. Eur J Dent 2016;10(3):361-365.

[5] Nikolaidis A, Vouzara T, Koulaouzidou E. Pit and fissure nanocomposite sealants reinforced with organically modified montmorillonite: A study of their mechanical properties, surface roughness and color stability. Dent Mater 2020;1:1-11.

[6] Ruschel VC, Bon VS, Baratieri LN, Maia HP. Effect of Surface Sealants and Polishing Time on Composite Surface Roughness and Microhardness. Oper Dent 2018;43(4):408-415.

[7] Hepdeniz OK, Temel UB, Ugurlu M, Koskan O. The effect of surface sealants with different filler content on microleakage of Class V resin composite restorations. Eur J Dent 2016;10(2):163-169.

[8] Tekçe N, Pala K, Tuncer S, Demirci M. The effect of surface sealant application and accelerated aging on posterior restorative surfaces: An SEM and AFM study. Dent Mater 2017;36(2):182-189.

[9] Spina DR., Grossi JR, Cunali RS, Filho FB, da Cunha LF, Gonzaga CC, Correr CM. Evaluation of Discoloration Removal by Polishing Resin Composites Submitted to Staining in Different Drink Solutions. Int Sch Res Notices 2015;2015:1-5.

[10] Pustina-Krasniqi T, Shala K, Staka G, Bicaj T, Ahmedi E, Dula L. Lightness, chroma, and hue distributions in natural teeth measured by a spectrophotometer. Eur J Dent 2017;11(1):36-40.

[11] He WH, Park CJ, Byun S, Tan D, Lin CY, Chee W. Evaluating the relationship between tooth color and enamel thickness, using twin flash photography, cross-polarization photography, and spectrophotometer. J Esthet Restor Dent 2019;1:1-11.

[12] Lee YK. Comparison of CIELAB $\triangle E^{*}$ and CIEDE2000 colordifferences after polymerization and thermocycling of resin composites. Dent Mater 2005;21(7):678-682.

[13] Doray PG, Eldiwany MS, Powers JM. Effect of resin surface sealents on improvement of stain resistance for a composite provisional material. J Esthet Restor Dent 2003;15:244-249.

[14] Rajkumar K, Kumar S, Mahalaxmi S, Ragavi P, Mageshwaran TA. Colour stability of resin composites after emersing in coffee of different temperature - an in vitro study. SRM Univ J Dent Sci 2011;2(2):91-95.

[15] Hepdeniz OK, Temel UB, Ugurlu M, Koskan O. The effect of surface sealants with different filler content on microleakage of Class $\mathrm{V}$ resin composite restorations. Eur J Dent 2016;10(2):163-169.

[16] Tuncer S, Demirci M, Tiryaki M, Ünlü N, Uysal Ö. The effect of a modeling resin and thermocycling on the surface hardness, roughness, and color of different resin composites. J Esthet Restor Dent 2013;25(6):404-419. 
[17] Barutcigil Ç, Yildiz M. Intrinsic and extrinsic discoloration of dimethacrylate and silorane based composites. J Dent 2012;40(1):57-63.

[18] Bagheri R, Burrow MF, Tyas M. Influence of food-simulating solutions and surface finish on susceptibility to staining of aesthetic restorative materials. J Dent 2005;33(5):389-398.

[19] Khokhar ZA, Razzoog ME. Yaman P. Color stability of restorative resins. Quintessence Int 1991;22(9):733-777.

[20] Mundim FM, Garcia LDFR, Pires-de-Souza FDC. Effect of staining solutions and repolishing on color stability of direct composites. J Appl Oral Sci 2010;18(3):249-254.

[21] Guler AU, Yilmaz F, Kulunk T, Guler E, Kurt S. Effects of different drinks on stainability of resin composite provisional restorative materials. J Prosthet Dent 2005;94(2):118-124.

[22] Reddy PS, Tejaswi KL, Shetty S, Annapoorna BM, Pujari SC, Thippeswamy HM. Effects of commonly consumed beverages on surface roughness and color stability of the nano, microhybrid and hybrid composite resins: an in vitro study. J Contemp Dent Pract 2013;14(4):718-723.
[23] Nasim I, Neelakantan P, Sujeer R, Subbarao CV. Color stability of microfilled, micro-hybrid and nanocomposite resins-an in vitro study. J Dent 2010;38(2):137-142.

[24] Ardu S, Duc O, Di Bella E, Krejci I, Daher R. Color stability of different composite resins after polishing. Odontol 2018;106(3):328-333.

[25] Kalachandra S, Turner DT. Water sorption of polymethacrylate networks: bis-GMA/TEGDM copolymers. J Biomed Mater Res 1987;21:329-338.

[26] Şahin O, Dede DÖ, Köroğlu A, Yılmaz B. Influence of surface sealant agents on the surface roughness and color stability of artificial teeth. J Prosthet Dent 2015;114(1):130-137.

[27] Lu H, Roeder LB, Lei L, Powers JM. Effect of surface roughness on stain resistance of dental resin composites. J Esthet Restor Dent 2005;17:102-108.

[28] Corcodel N, Hassel AJ, Sen S, Saure D, Hum S, Rammelsberg $P$, Lux CJ, Zingler S. Effects of staining and polishing on different types of enamel surface sealants. J Esthet Restor Dent 2018;30(6):580-586.

How to cite this article: Korkut B. Colorimetric Assessment of Surface Sealants for Discoloration of a Nanofilled Resin Composite. Clin Exp Health Sci 2020; 10: 448-453. DOI: 10.33808/clinexphealthsci.808644 